\title{
Dextran sulfate inhibition on human gastric cancer cells invasion, migration and epithelial-mesenchymal transformation
}

\author{
YUANYI XU ${ }^{1 *}$, XIU JIN ${ }^{2 *}$, YUNNING HUANG ${ }^{3 *}$, JUAN WANG ${ }^{1}$, XIAOFEI WANG ${ }^{1}$ and HONGHONG WANG ${ }^{4}$ \\ ${ }^{1}$ Department of Pathology, Ningxia Medical University, Yinchuan, Ningxia Hui Autonomous Region 750001; \\ ${ }^{2}$ Department of Pathology, Affiliated Hospital of Jining Medical University, Jining, Shandong 272029; \\ Departments of ${ }^{3}$ Gastrointestinal Surgery and ${ }^{4}$ Pathology, Ningxia People's Hospital, \\ Yinchuan, Ningxia Hui Autonomous Region 750021, P.R. China
}

Received August 10, 2017; Accepted March 2, 2018

DOI: $10.3892 / \mathrm{ol} .2018 .9251$

\begin{abstract}
The objective of the present study was to observe the influence of dextran sulfate (DS) on the proliferation, invasion and migration of AGS, BGC-23, GES-1, MGC-803 and SGC-7901 cells. Additionally, the possible inhibition mechanism of DS on BGC-823 cells epithelial-mesenchymal transition (EMT) was explored. The cells in the control and experimental group were treated with PBS and DS respectively. The effect of DS on the invasion and migration of these five types of cells were investigated using Transwell invasion and migration assays. Immunocytochemistry, western blotting and reverse transcription-polymerase chain reaction (RT-PCR) assays were used to measure gene and protein expression of hypoxia-inducible factor $1 \alpha$ (HIF1-a) and EMT associated factors [Twist, E-cadherin, $\mathrm{N}$-cadherin and $\beta$-catenin] of BGC-823 cells. According to the results of CCK-8, DS significantly decreased the proliferation of AGS, SGC-7901 and BGC-823 cells to different extents, but there were no notable differences for MGC-803 cells. Transwell migration and invasion results demonstrated that, compared with the control group, DS reduced the migration and invasion of every types of cells to different extents, and the inhibition to BGC-823 cells invasion is the most notably. Immunofluorescence, RT-PCR and western blot analysis results indicated that HIF-1 $\alpha$, Twist and $\mathrm{N}$-cad expressions levels
\end{abstract}

Correspondence to: Dr Yunning Huang, Department of Gastrointestinal Surgery, Ningxia People's Hospital, 301 Zhenyuan North Street, Yinchuan, Ningxia Hui Autonomous Region 750021, P.R. China

E-mail:nxhyncc@126.com

Mrs. Yuanyi Xu, Department of Pathology, Ningxia Medical University, 1160 Shenli South Street, Yinchuan, Ningxia Hui Autonomous Region 750001, P.R. China

E-mail: nxxyy@hotmail.com

${ }^{*}$ Contributed equally

Key words: dextran sulfate, human gastric cancer cells, metastasis, epithelial-mesenchymal transition had different degrees of reduction in the experimental group following DS treatment; however, the expression level of E-cad had increased. In conclusion, DS inhibited the proliferation of AGS, BGC-823, SGC-7901 and GES-1 cells, the inhibition degree may be associated with the differentiation degree of every cancer cell, the higher the differentiation degree, the stronger the inhibition. DS inhibited migration and invasion of the five types of gastric cancer cells in different degree. DS may inhibit EMT of BGC-823 by inhibiting Wnt signaling.

\section{Introduction}

Morbidity rate and mortality rate of gastric cancer are the highest for digestive tract tumors globally, and the results indicate a younger trend $(1,2)$. Drug therapy is the main treatment option for intra-abdominal planting transfer of gastric cancer; however, thus far the curative effect is still not sufficient. Dextran sulfate (DS) is a macromolecule Dextran with a molecular weight of 500,000. Previous studies have demonstrated that DS can inhibit cancer cells adhesion, cell cycle progression and gene expression $(3,4)$, DS can reduce of expression of gastric cancer cells in terms of vascular endothelial growth factor (VEGF) and integrin $\beta 1$ in vivo and in vitro, and there was a significant inhibitory effect on gastric cancer cell adhesion and angiogenesis (5), but the inhibiting mechanism of DS on the metastasis of gastric cancer still requires investigating.

Peritoneal transfer of gastric cancer is a multi-factor, multi-phase continuous complex process. In this process, epithelial-mesenchymal transformation (EMT) serves a key role in promoting the invasion and metastasis of tumor cells (6). EMT mechanism is a complicated process, and hypoxia is one of the most important initiating factors to EMT (7), In a hypoxic microenvironment, cancer cells adapt to the hypoxic microenvironment through highly expressing hypoxia-inducible factor $1 \alpha(\mathrm{HIF}-1 \alpha)$ (8), HIF-1 $\alpha$ high expression then promote: i) EMT markers, including Twist, E-cad and N-cad; ii) the regulation of angiogenesis, including vascular endothelial growth factor (VEGF); iii) cell adhesion, including ITG $\beta 1(9,10)$. At present, the examination of EMT, invasion and migration mechanisms is the notable basis for the development in the diagnosis and treatment of gastric 
cancer, and additionally helps to provide potential intervention targets for the treatment of a malignant tumor (11). The present study investigated the inhibiting effect of DS on EMT by measuring the expression of EMT associated factors [Twist, E-cadherin (E-cad), N-cadherin (N-cad) and $\beta$-catenin], proliferation, invasion and migration of 4 types of different degree of differentiate gastric cancer cells in the process of peritoneal metastasis, and then suggested a theoretical basis for discovering a potential drug in the treatment of peritoneal metastasis of gastric cancer.

\section{Materials and methods}

Gastric cancer cell lines. Poorly differentiation gastric cancer cells (BGC-823) were purchased from Cobioer; Nanjing Kesheng Biotechnology Co., Ltd. (Nanjing, China) High differentiation gastric cancer cells AGS, undifferentiated gastric cancer cells MGC-803, lymph node metastasis gastric cancer cells SGC-7901 and normal gastric mucosa epithelial cells GES-1, were donated from The Life Science Laboratory of Shanghai East China Normal University (Shanghai, China).

The experimental drugs and antibodies. DS, with a molecular weight $\sim 500,000$, was purchased from Sigma-Aldrich (Merck KGaA, Darmstadt, Germany). A rabbit anti-human anti-HIF-1 $\alpha$ monoclonal antibody (Wuhan Sanying Biotechnology, Wuhan, China; cat. no. 20960-1-AP; dilution, 1:1,500), rabbit anti-human E-cad Polyclonal Antibody (cat. no. E-AB-31261; dilution, 1:200) and rabbit anti-human N-cad Polyclonal Antibody (cat. no. E-AB-32170; dilution, 1:1,000), were purchased from the Elabscience Biotechnology Co., Ltd., Wuhan, China. Anti-Twist antibody (cat. no. ab50581; dilution, 1:1,000) was purchased from Abcam (Cambridge, UK). A goat anti-rabbit (cat. no. BA1003; dilution 1:5,000) biotin-conjugated immunoglobulin IgG (Wuhan Boster Biological Technology Co., Ltd.) was used to stain the primary antibody.

Cell culture. A total of five types of cells were cultured in complete medium (Beijing Zhongshan Jinqiao Biotechnology Co., Ltd., Beijing, China) in a sterile conditions at a constant temperature of $37^{\circ} \mathrm{C}$ and $5 \% \mathrm{CO}_{2}$ until the logarithmic phase. Then, cells were placed into a $60 \mathrm{~mm}$ petri dish. The control group and experimental group were exposed to the same amount of PBS and DS (final concentration of $0.3 \%$ ), respectively. Cells were collected for inspection at six time points $(0,6,12,24,36$ and $48 \mathrm{~h}$ after culture). BGC-823 cells were cultured in a hypoxia incubator at $37^{\circ} \mathrm{C}$ with $5 \% \quad \mathrm{CO}_{2}$ and $1 \% \mathrm{O}_{2}$, and cells collected for inspection at six time points $(0$, $6,12,24,36$ and $48 \mathrm{~h}$ after culture). The absence of a positive control group is a limitation of the present study.

Cell Counting kit-8 (CCK-8) assays to detect cell proliferation. Adjusting the cell suspension density to $2.5 \times 10^{4} / \mathrm{ml}$ in RPMI-1,640 medium (Hyclone; GE Healthcare Life Sciences, Logan, UT, USA), cells were placed in six 96-well plates at $200 \mu \mathrm{l} /$ well, and the culture was placed in the incubator at $37^{\circ} \mathrm{C}$ and $5 \% \mathrm{CO}_{2}$ for $\sim 12 \mathrm{~h}$. When the cells attached to the side of the well, the experimental group was treated with the culture medium containing $0.3 \% \mathrm{DS}$; furthermore, the control group was treated with the culture medium containing the same amount of
PBS. Following 0, 6, 12, 24, 36 and 48 h, $10 \mu \mathrm{l} \mathrm{CCK-8} \mathrm{fluid} \mathrm{was}$ added into each well and then placed back into the incubator to develop for $4 \mathrm{~h}$ at $37^{\circ} \mathrm{C}$ and $5 \% \mathrm{CO}_{2}$. The absorbance (OD) value at $450 \mathrm{~nm}$ for each well was tested by using a microplate reader. Finally, an average value of the five complex wells for each group.

Transwell cell invasion and migration assays. Matrigel was placed into the upper layer of the Transwell chamber and following solidification, the cell density was adjusted to $1 \times 10^{5} / \mathrm{ml}$. A total of $200 \mu \mathrm{l}$ cells in serum-free medium (Hangzhou Sijiqing Biological Engineering Materials Co., Ltd., Hangzhou, China) were placed into the upper chamber of an insert. Then, $500 \mu 1$ medium containing FBS and DS was added to the lower chamber of the experimental group, and $500 \mu \mathrm{l}$ medium containing FBS and PBS was added to the lower chamber of the control group. After incubation for $24 \mathrm{~h}$ at $37^{\circ} \mathrm{C}$, cells that had invaded and migrated through the membrane could be observed by staining with $0.1 \%$ crystal violet staining solution for $20 \mathrm{~min}$ at $37^{\circ} \mathrm{C}$, and 5-10 visual fields were taken for counting the average number of the cells using an inverted microscope (Olympus Corporation, Tokyo, Japan).

Cell immunofluorescence staining. Treated cells were fixed for $14 \mathrm{~min}$ with cold $4 \%$ polyphosphate formaldehyde, and the fixed cells were permeabilized with $0.1 \%$ Triton X-100 (Beijing Zhongshan Jinqiao Biotechnology Co., Ltd.) for 30 min, 3 times, and then blocked with $10 \%$ goat serum (Beijing Zhongshan Jinqiao Biotechnology Co., Ltd.), for $30 \mathrm{~min}$. Following incubation overnight at $4^{\circ} \mathrm{C}$ with primary antibodies (twist, E-cad, N-cad; dilution 1:100), FITC marked fluorescence second antibody (1:200) was added to the cells. Following an incubation at $37^{\circ} \mathrm{C}$ for $0.5 \mathrm{~h}$, DAPI was added in the dark, the film was sealed and observed using a fluorescence microscope with a magnification of x100. Cumulative density demonstrated the expression quantity of the factors, which was analyzed by Student's t-test using GraphPad Prism 7 plotting software (GraphPad Software, Inc., La Jolla, CA, USA).

Detection of HIF-1 $\alpha$ and EMT associated factors mRNA using reverse transcription-polymerase chain reaction $(R T-P C R)$. The primers used were: HIF-1 $\alpha$ forward, 5'-GAA AGCGCAAGTCTTCAAAG-3' and reverse, 5'-TGGGTA GGAGATGGAGATGC-3'; Twist forward, 5'-AATTGGGAT GCATTCGAGTCTGTAA-3' and reverse, 5'-TTCTGTCCG ATGTCACTGCTGTC-3'; E-cad forward, 5'-TACACTGCCC AGGAGCCAGA-3' and reverse, 5'-TGGCACCAGTGTCCG GATTA-3'; N-cad forward, 5'-ACCTGAACGACTGGGGGC CA-3' and reverse, 5'-TGCCAAAGCCTCCAGCAAGCA-3'; and GAPDH (internal control) forward, 5'-CAAGGTCAT CCATGACAACTTTG-3' and reverse, 5'-GTCCACCACCCT GTTGCTGTAG-3'. The primers were synthesized by Sangon Biotech Co., Ltd., Shanghai, China. Treated cells at the time points of 2, 8, 12 and $24 \mathrm{~h}$ were lysed, and total RNA was extracted and reverse transcribed by using Total mRNA extraction kit (Omega Bio-Tek, Inc., Norcross, GA, USA) and reverse transcription kit (Fermentas; Thermo Fisher Scientific, Inc., Waltham, MA, USA) following the manufacturer's protocol. Following the denaturation of cDNA $(2 \mu \mathrm{l})$ at $94^{\circ} \mathrm{C}$ for $3 \mathrm{~min}$, temperature cycling (30 cycles) was performed as follows: $94^{\circ} \mathrm{C}$ for $45 \mathrm{sec}, 30 \mathrm{sec}$ annealing step at $58^{\circ} \mathrm{C}$ (for GAPDH, 

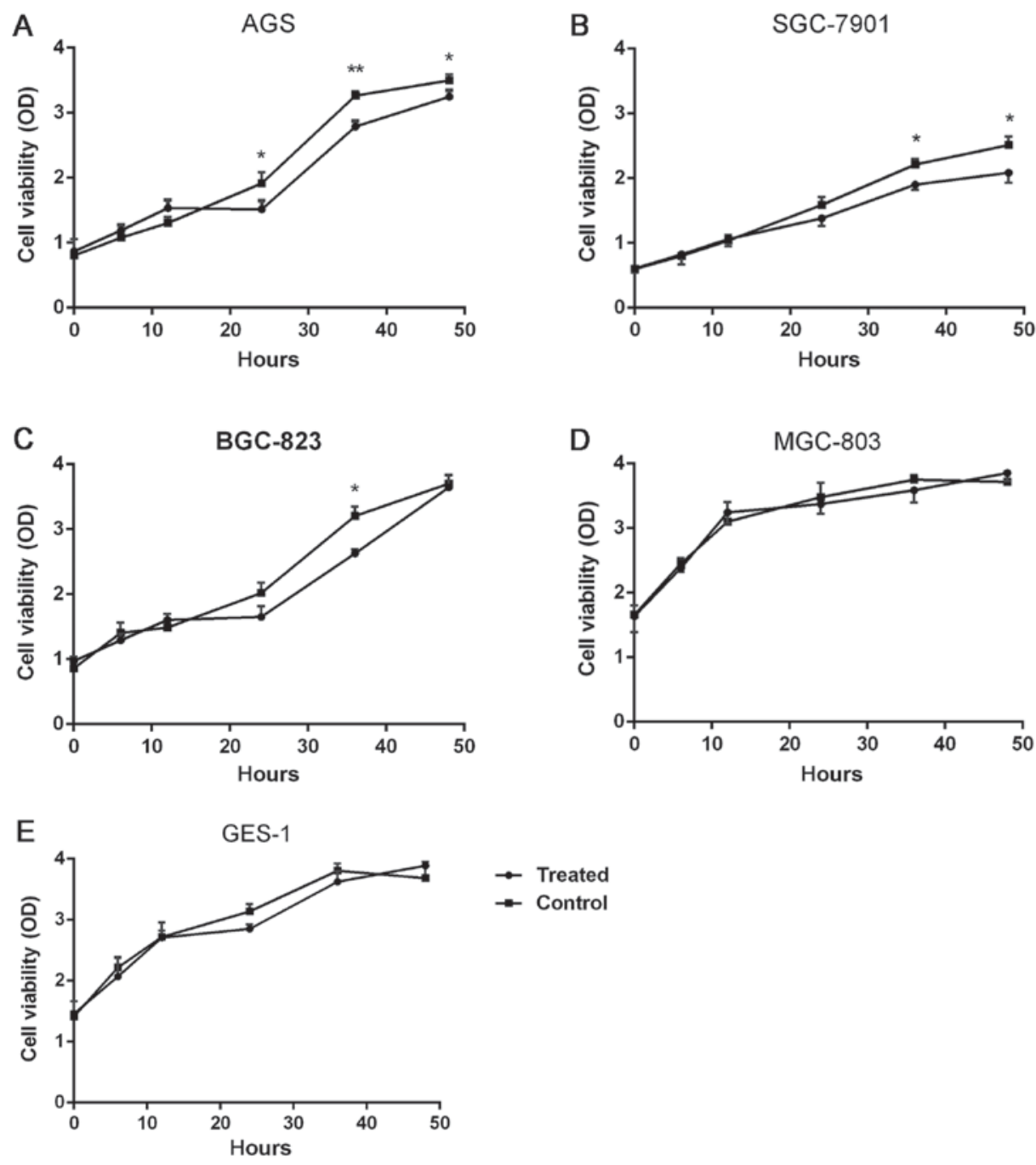

Figure 1. Dextran sulfate impact on the proliferation of five types of cells. The Cell Counting kit-8 detection of (A) AGS, (B) SGC-7901, (C) BGC-823, (D) MGC-803 and (E) GES-1. ${ }^{*} \mathrm{P}<0.05,{ }^{* *} \mathrm{P}<0.01$ vs. the control. OD, optical density.

Twist, E-cad and $\mathrm{N}$-cad) and a $30 \mathrm{sec}$ elongation step at $72^{\circ} \mathrm{C}$. Temperature cycling was concluded with a final elongation step for $5 \mathrm{~min}$ at $72^{\circ} \mathrm{C}$. This procedure was followed by grey value analysis using Quantity one version 4.0 (Bio-Rad Laboratories), the purpose/internal relative grayscale value indicated the corresponding mRNA expression level. The mRNA expression level of the cells in the experimental group was compared with the control group at the time points of $2,8,12$ and $24 \mathrm{~h}$, respectively.

Western blot detection of HIF-1 $\alpha$ and EMT associated factor protein expression. Cultured BGC823 cells were washed by ice-cold PBS three times. Cell lysis solution (Nanjing KeyGen Biotech Co., Ltd, Nanjing, China) was added, the dish agitated for $30 \mathrm{~min}$, centrifuged at $12,000 \mathrm{xg}$ at $4^{\circ} \mathrm{C}$ for $15 \mathrm{~min}$ and the supernatant removed. Detection of protein concentration was conducted using BCA protein quantitative kits. Protein extracts $(20 \mu \mathrm{g})$ were separated by $8-10 \%$ SDS polyacrylamide gel electrophoresis, then transferred to nitrocellulose membranes and blocked by $10 \%$ skim milk for $1.5 \mathrm{~h}$ at $4^{\circ} \mathrm{C}$, then incubated by the corresponding antibody at $4^{\circ} \mathrm{C}$ overnight (HIF-1 $\alpha, 1: 1,000$; Twist, 1:500; E-cad, 1:200; N-cad, 1:1,000; and $\beta$-actin, 1:1,500), $\beta$-actin was used as an internal control. The second antibody incubation was at room temperature for $1 \mathrm{~h}$, followed by ECL chemiluminescence reagent chromogenic (cat. no. MA02454; Thermo Fisher Scientific, Inc.) for $1 \mathrm{~min}$ and exposure in an Amersham Imager 600 instrument (GE Healthcare Life Sciences, Little Chalfont, UK). This was followed with grey value analysis with Quantity one, the relative grayscale value indicating the corresponding protein expression level.

Statistical analysis. All the experiments were performed in triplicate. SPSS version 17.0 (SPSS, Inc., Chicago, IL, USA) was used to perform the statistical analyses. Data are expressed as the mean \pm standard deviation. The comparison of two groups was performed using the Students' t-test. One-way analysis of variance was used in the comparison of multiple groups followed by Dunnett's post-hoc test. $\mathrm{P}<0.05$ was considered to indicate a statistically significant difference.

\section{Results}

DS impact on the proliferation of five types of cells. There were five types of cells examined at six time points, $0,6,12$, 24,36 and $48 \mathrm{~h}$. These results demonstrated that for the AGS cells at $24 \mathrm{~h}(\mathrm{P}=0.032,21.1 \%), 36 \mathrm{~h}(\mathrm{P}=0.0076,15.4 \%)$ and 

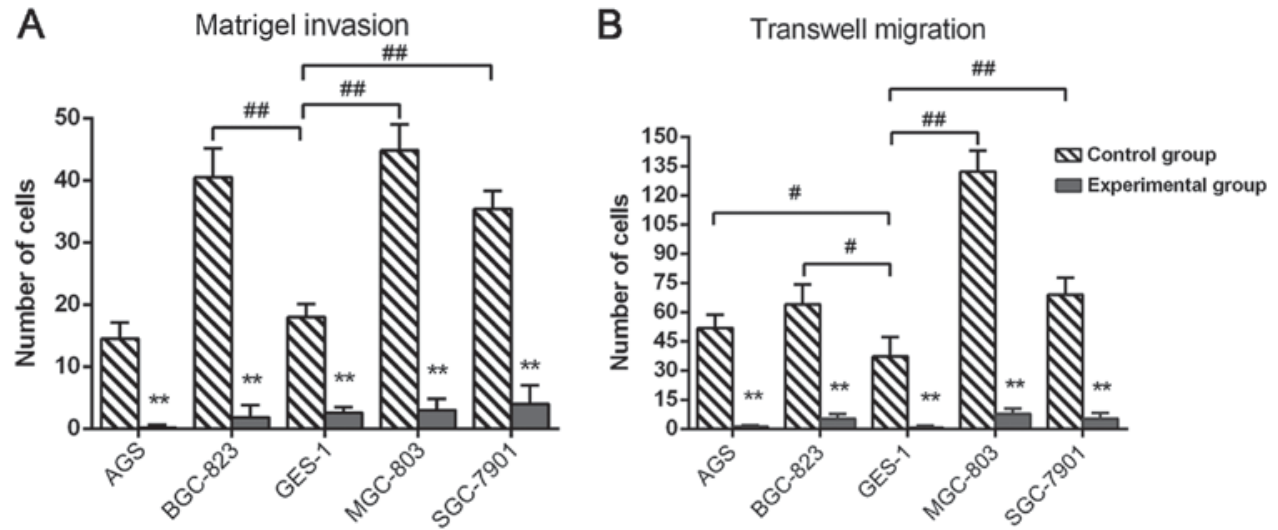

Figure 2. Dextran sulfate inhibits the invasive ability and migration ability of gastric cells. (A) Matrigel invasion and (B) Transwell migration assays for the number cells that passed through the membrane, ${ }^{* *} \mathrm{P}<0.01$ vs. control group. ${ }^{~} \mathrm{P}<0.05$ and ${ }^{\# \#} \mathrm{P}<0.01$.

$48 \mathrm{~h}(\mathrm{P}=0.041,7.5 \%)$, the cell proliferation in the experimental group was significantly reduced, compared with the control group, as depicted in Fig. 1A. The proliferation of SGC-7901 cells was significantly inhibited by DS, as depicted in Fig. 1B, at $36 \mathrm{~h}(\mathrm{P}=0.036,20 \%)$ and $48 \mathrm{~h}(\mathrm{P}=0.027,19.5 \%)$ For BGC- 823 cells, DS significantly reduced the proliferation of the cells at 36 h, (P=0.043, 18.8\%), as depicted in Fig. 1C. For MGC-803 and GES-1 cells, no significant difference in cell proliferation was observed in the experimental group and control group at $0,6,12,24,36$ and $48 \mathrm{~h}$, as depicted in Fig. 1D and E.

DS inhibits the invasive ability and migration ability of gastric cells. In the control group $24 \mathrm{~h}$ after growing on Matrigel membrane, the number of the invaded cancer cells BGC-823 $(\mathrm{P}=0.0042)$, MGC-803 ( $\mathrm{P}=0.0098)$ and SGC-7901 ( $\mathrm{P}=0.0079)$ was significantly increased compared with GES-1 cells, no significant difference was observed between AGS and GES-1 cells; in the control group, the average number of invaded cancer cells was highest in the MGC-803 cell line, followed by BGC-823, SGC-7901 and AGS in turn. Compared with the control group, the number of invasive cancer cells in the experimental group was significantly reduced by DS, and BGC-823 cells (inhibition rate 95\%, $\mathrm{P}=0.0021$ ) had the most notable inhibition, followed by AGS $(92.8 \%, \mathrm{P}=0.0033)$, MGC- 803 (90.1\%, P=0.0042), SGC-7901 (87.8\%, P=0.0057) and GES-1 cells $(72.3 \%, \mathrm{P}=0.0076<0.01)$, as depicted in Fig. $2 \mathrm{~A}$. In the control group, following $24 \mathrm{~h}$ growth on a Transwell membrane, the number of migrated gastric cancer cell lines AGS ( $\mathrm{P}=0.036), \mathrm{BGC}-823(\mathrm{P}=0.29)$, MGC-803 $(\mathrm{P}=0.0075)$ and SGC-7901 $(\mathrm{P}=0.0053)$ was significantly greater, compared with GES-1 cell; in the control group, the average number of migrated cells was highest in MGC- 803 cells, followed by BGC-823, SGC-7901 and AGS. In the experimental group, DS significantly reduced all the five cell numbers on the membrane compared with control group, and the migration inhibition of AGS cells (inhibition rate $96 \%, \mathrm{P}=0.00062$ ) is the most notable, followed by SGC-7901 (94.1\%, P=0.0074), MGC-803 (91.2\%, $\mathrm{P}=0.0083)$, BGC-823 (90.5\%, $\mathrm{P}=0.0087)$ and GES-1 cells (84.3\%, $\mathrm{P}=0.0091)$, as depicted in Fig. 2B.

Immunofluorescence staining of HIF-1 $\alpha$ and EMT associated factors in BGC-823 cells. Following prolonged hypoxia, in the control group, the expression of HIF-1 $\alpha$, N-cad gradually increased at 2, 8, 12 and $24 \mathrm{~h}$. In the experimental group, the HIF-1 $\alpha(\mathrm{P}=0.022)$ and $\mathrm{N}-\mathrm{cad}(\mathrm{P}=0.031)$ expression levels were significant reduced, compared with the control group; E-cad expression in control group cells gradually reduced, with a tendency to slow approaching $24 \mathrm{~h}$. At 8, 12 and $24 \mathrm{~h}$, the DS significantly slowed down in reducing E-cad expression. $\beta$-catenin expression in the control group expression gradually increased. In the cytoplasm and nuclei, the expression in the experimental group in the nucleus and cytoplasm was less, compared with the increase in the control group. The DS at 2, 8,12 and $24 \mathrm{~h}$ significantly reduced the experimental $\beta$-catenin expression.

Following prolonged culture in oxygen, the control group HIF- $1 \alpha$ expression increased gradually after $8 \mathrm{~h}$, and at 8,12 and $24 \mathrm{~h}$, the expression significantly decreased in the experimental group, compared with the control group. In the control cells after $12 \mathrm{~h}$, E-cad had a significantly lower expression, $\mathrm{N}$-cad was significantly upregulated, DS significantly slowed down in the reduction of E-cad, and there was an increase of $\mathrm{N}$-cad at 12 and $24 \mathrm{~h}$. $\beta$-catenin expression in control cells increased gradually after $8 \mathrm{~h}$, with a gradual increase in the cytoplasm and nuclei, and relative decrease in the nucleus and cytoplasm of experimental group, at 8, 12 and $24 \mathrm{~h}$. Additionally, DS significantly reduced the $\beta$-catenin expression of the BGC-823 cells, as depicted in Fig. 3 .

RT-PCR detection of HIF-1 $\alpha$ and EMT associated factors $m R N A$ expression in BGC-823 cells. When hypoxia time was extended, in the control group the cell HIF-1 $\alpha$, Twist and $\mathrm{N}$-cad, $\beta$-catenin mRNA level gradually increased, whereas E-cad mRNA level gradually reduced. At 2, 8, 12 and $24 \mathrm{~h}$, the mRNA level of HIF-1 $\alpha$ and Twist in the experimental group was significantly lower, compared with the control group, and the experimental group E-cad mRNA level was significantly increased, compared with the control group. At 2, 8 and $12 \mathrm{~h}$, the N-cad and $\beta$-catenin mRNA level of the experimental group increased significantly, compared with the control group.

Following prolonged culture in oxygen, in the control group cells the mRNA level of HIF-1 $\alpha$, Twist, N-cad and $\beta$-catenin all demonstrated an increasing trend, whereas the E-cad mRNA level gradually reduced. At 8, 12 and $24 \mathrm{~h}$, the 

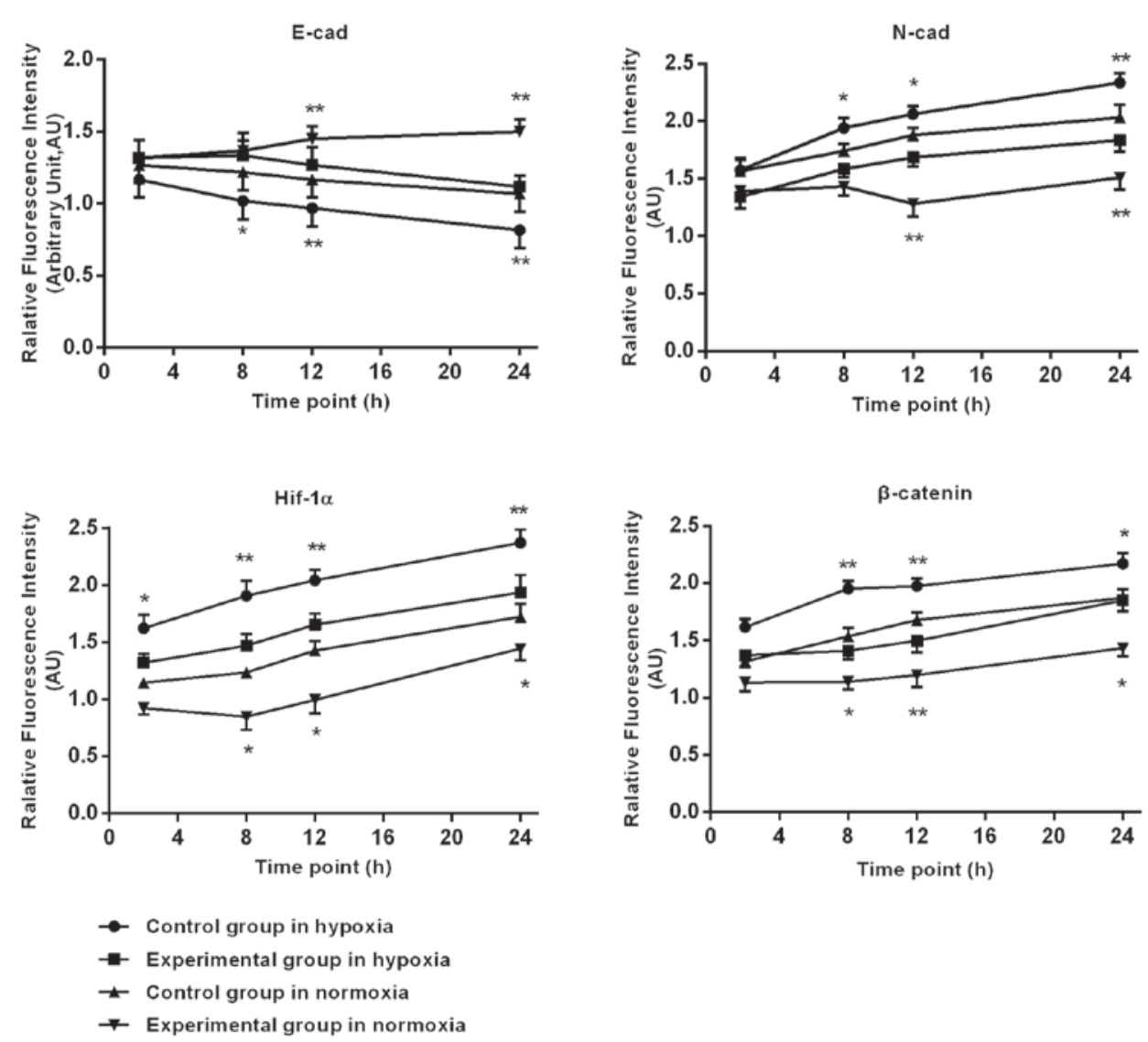

Figure 3. Immunofluorescence staining of HIF-1 $\alpha$ and epithelial-mesenchymal transition associated factors in BGC-823 cells. Statistical analysis of immunofluorescence staining of HIF-1 $\alpha, \beta$-catenin, E-cad and N-cad in BGC-823 cells. "P<0.05 and ${ }^{* *} \mathrm{P}<0.01$, experimental vs. control group. HIF-1 $\alpha$, hypoxia-inducible factor $1 \alpha$; E-cad, E-cadherin; $\mathrm{N}$-cad, $\mathrm{N}$-cadherin; $\beta$-cat, $\beta$-catenin.

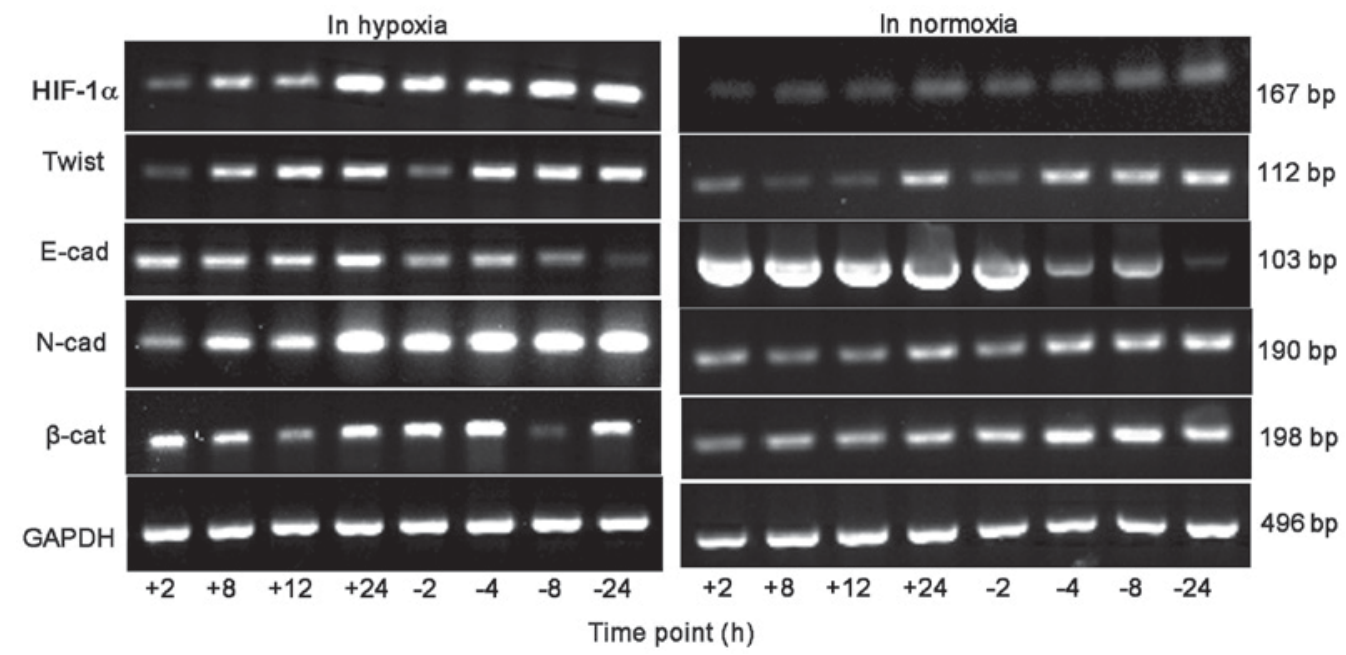

Figure 4. Reverse transcription-quantitative polymerase chain reaction detection of HIF-1 $\alpha$ and epithelial-mesenchymal transition associated factors mRNA expression in BGC-823 cells. Electrophoretic band of HIF- $1^{\alpha}$ associated factors $\beta$-catenin, E-cad and N-cad mRNA in hypoxia and normoxia in BGC-823 cells. +, time in experimental group; -, time in control group. HIF-1 $\alpha$, hypoxia-inducible factor $1 \alpha$; E-cad, E-cadherin; N-cad, N-cadherin; $\beta$-cat, $\beta$-catenin.

mRNA levels of HIF-1 $\alpha$, Twist and $\beta$-catenin in the experimental group were significantly decreased, compared with the control group, and the experimental group E-cad mRNA level was significantly increased, compared with the control group. At 12 and $24 \mathrm{~h}$, the N-cad mRNA level of the experimental group was significantly increased, compared with the control group, as depicted in Figs. 4 and 5.
Western blot detection of EMT associated factors protein expression in BGC-823 cells. In hypoxia environment, as the duration of hypoxia increased, HIF-1 $\alpha$, Twist, N-cad and $\beta$-catenin expression gradually increased in the control group, and HIF- $1 \alpha, \mathrm{N}$-cad and $\beta$-catenin expression of the experimental group was significantly lower, compared with the control group, at 2, 8, 12 and $24 \mathrm{~h}$. At 8, 12 and $24 \mathrm{~h}$, Twist 

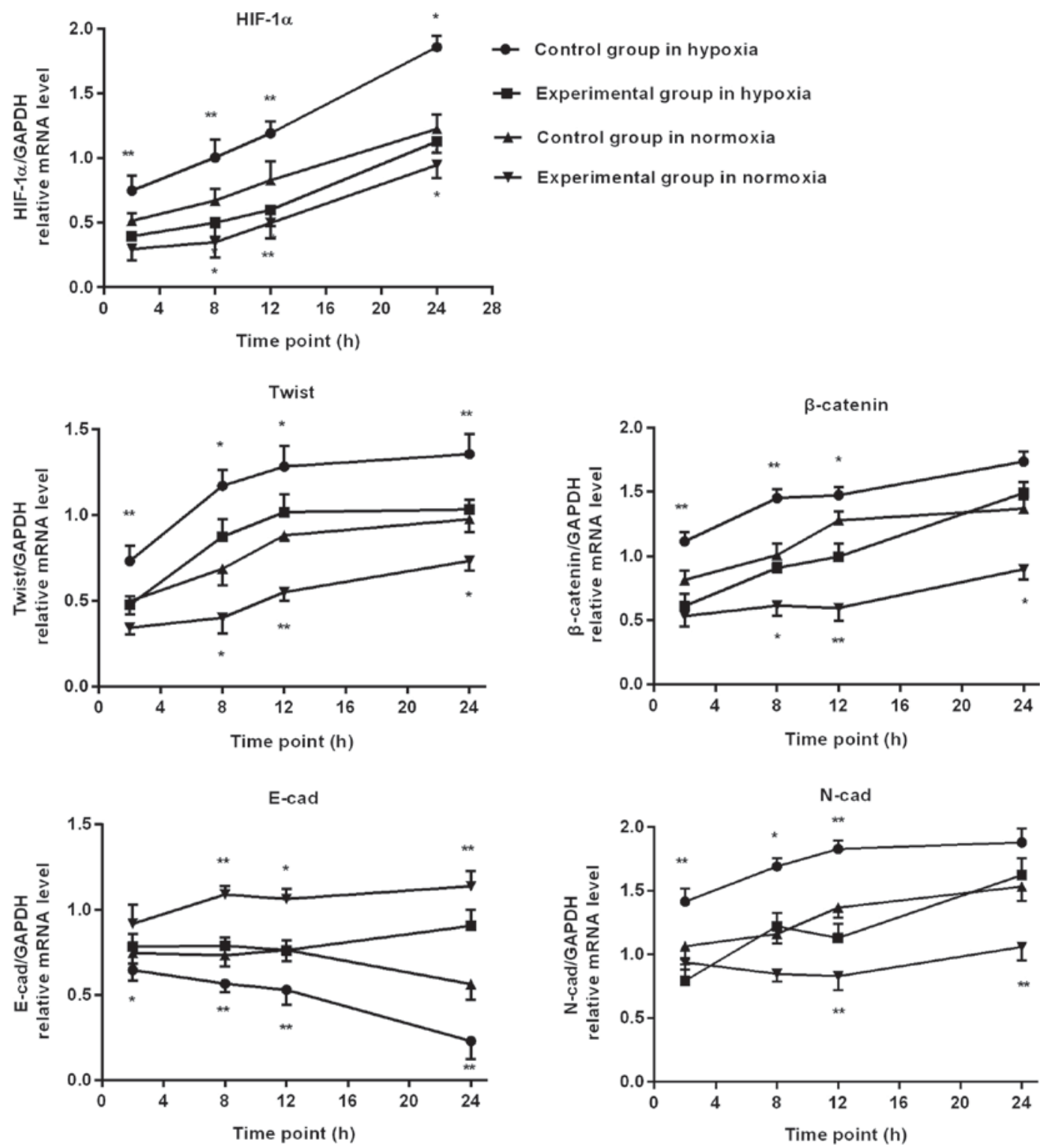

Figure 5. Reverse transcription-polymerase chain reaction statistics of HIF- $1 \alpha$, Twist, $\beta$-catenin, E-cad and N-cad mRNA expression level. " $\mathrm{P}<0.05$ and ${ }^{* *} \mathrm{P}<0.01$ experimental vs. control group. HIF-1 $\alpha$, hypoxia-inducible factor $1 \alpha$; E-cad, E-cadherin; N-cad, N-cadherin.

expression of the experimental group was significantly lower, compared with the control group, and the expression of E-cad increased significantly, compared with the control group.

In normoxia environment, as the duration of normoxia increased, in the control group HIF- $\alpha$, Twist, $\beta$-catenin and $\mathrm{N}$-cad expression gradually increased, E-cad decreased at the time point of $12 \mathrm{~h}$. At 8, 12 and $24 \mathrm{~h}, \mathrm{HIF}-1 \alpha$, Twist and $\beta$-catenin expression in the experimental group was significantly lower, compared with the control group. At 12 and $24 \mathrm{~h}$, the experimental group $\mathrm{N}$-cad expression was significantly lower than the control group, and the expression of E-cad increased significantly, compared with the control group, as depicted in Figs. 6 and 7.

\section{Discussion}

The prevention and treatment of proliferation, invasion and metastasis of gastric cancer is one of the problems that require further study.
The present study used the gastric cancer cell lines AGS, BGC-823, MGC-803 and SGC-803 and normal gastric mucosa cells GES-1 to detect the effects of DS on proliferation, invasion and migration. The cell lines MGC-803 (undifferentiated), BGC-823 (low differentiation) and AGS (high differentiated) are all derived from gastric cancer tumors, and the SGC-7901 cell line was derived from lymph node metastasis (moderately differentiated). GES-1 is derived from human fetal gastric mucosa epithelial cells (immortalized) (12).

One in vitro study has demonstrated that DS can inhibit cancer cell adhesion, cell cycle progression and gene expression (3). In the present study, using a CCK-8 proliferation test, it was determined that at 6 and $12 \mathrm{~h}$ DS had no notable inhibitory effect on the proliferation of five types of cells. At $24 \mathrm{~h}$, DS inhibited AGS cell proliferation by $21.1 \%$, and DS reduced the proliferation of AGS, SGC7901 and BGC-823 cells by $15.4,18.8$ and $20 \%$, respectively at 36 h. At 48 h, DS reduced the proliferation of AGS and SGC-7901 cells by 19.5 and $7.5 \%$, respectively, and within 48 h, DS has no notable 


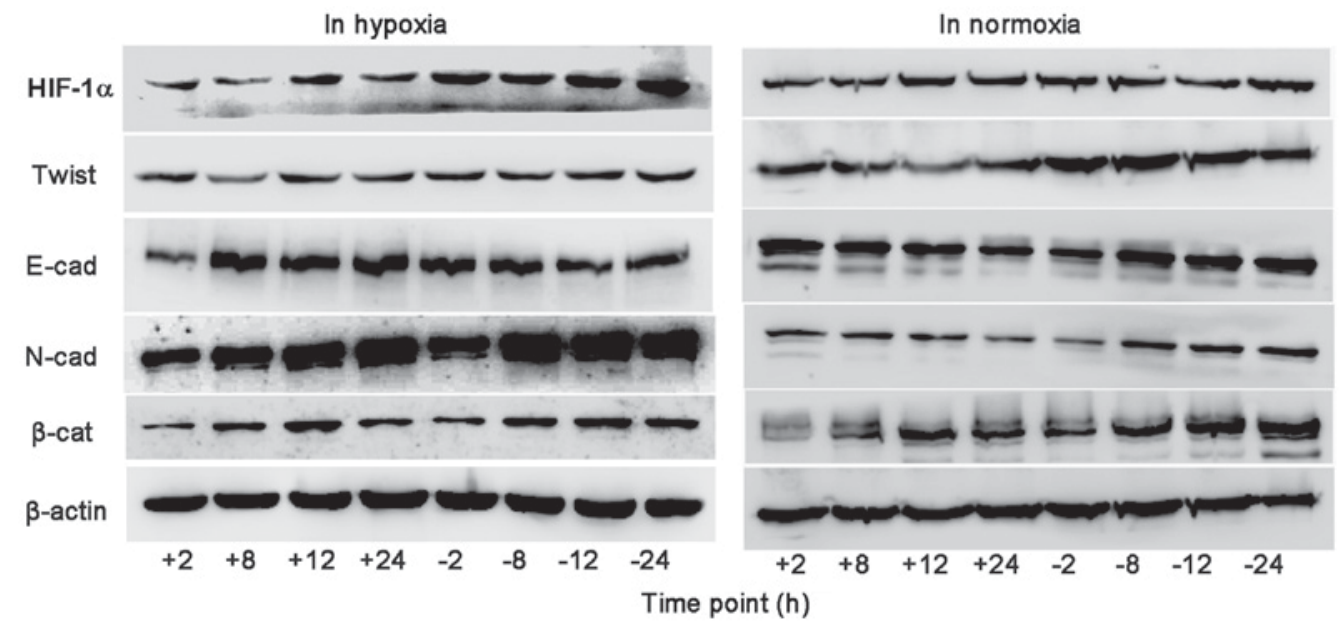

Figure 6. Western blot detection of epithelial-mesenchymal transition associated factors protein expression in BGC-823 cells. Electrophoretic band of epithelial-mesenchymal transition-associated factors Twist, $\beta$-catenin, E-cad and N-cad protein expression in hypoxia and in normoxia in BGC-823 cells. HIF-1 $\alpha$, hypoxia-inducible factor $1 \alpha$; E-cad, E-cadherin; N-cad, $N$-cadherin; $\beta$-cat, $\beta$-catenin.
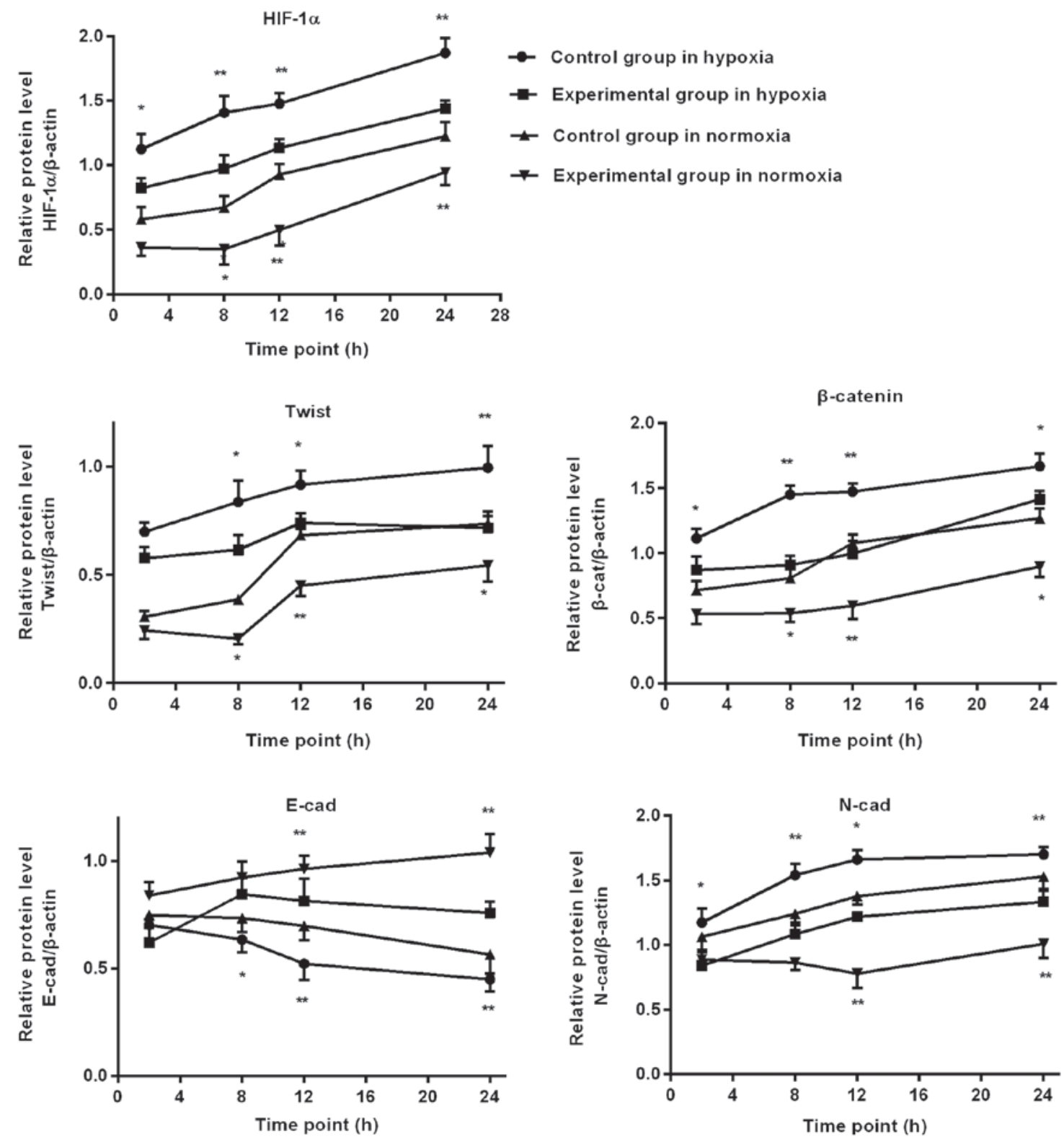

Figure 7. Western blot statistics of epithelial-mesenchymal transition-associated factors Twist, $\beta$-catenin, E-cad and $\mathrm{N}$-cad protein expression level in hypoxia and in normoxia. " $\mathrm{P}<0.05$ and ${ }^{* *} \mathrm{P}<0.01$ experimental vs. control group. HIF-1 $\alpha$, hypoxia-inducible factor $1 \alpha$; E-cad, E-cadherin; $\mathrm{N}$-cad, $\mathrm{N}$-cadherin; $\beta$-cat, $\beta$-catenin. 
inhibitory effect on MGC-803 cells. It may be safely concluded that DS inhibited the highly differentiated gastric cancer cell AGS most, as the reduction of the AGS cell number was the most significant, whilst it had no significant proliferation inhibition effect on the undifferentiated gastric cancer cell line MGC-803; Therefore, it was speculated that gastric cancer cell proliferation inhibition by DS may be associated with differentiation degree.

Tumor cells with different degrees of differentiation have varying invasion and migration abilities (13). Research has indicated that gastric cancer cell line BGC-823 has an improved invasion ability, compared with the cell lines AGS, SGC-7901 and GES-1 (14). The Transwell migration experiment in the present study demonstrated that migrated numbers of the four gastric cancer cell lines were notably more than the GES-1, the four gastric cancer cells lines had an improved ability to migrate, compared with the than normal gastric mucosa cells. Calculating the average gastric cancer cells that migrated, the undifferentiated gastric cancer cells MGC-803 had the highest, and then, in turn, the low differentiated gastric cancer cells BGC-823, moderately differentiated gastric cancer cells SGC-7901 and high differentiated gastric cancer cell AGS. This demonstrated that the lower degree of differentiation, the more cells that migrated across the membrane, indicating an improved migration ability. Significantly, DS reduced all five cell lines migration count, compared with the control group. The cell line with the most notable inhibitory effect was AGS, followed by SGC-7901, MGC-803, BGC-823 and GES-1. So it was speculated that DS may have an inhibitory effect on migration ability of all five types of cell lines, which may be associated with the degree of differentiation.

The primary difference between the invasion and migration experiment was the layer of Matrigel. Cells must penetrate the matrix firstly, then through the chamber on the underside of the membrane filter. The invasion experiment indicated that the average invaded cells was greatest for the undifferentiated MGC-803 gastric cancer cells, and then, in turn, the poorly differentiated gastric cancer cells BGC-823, moderately differentiated gastric cancer cells SGC-7901 and high differentiated gastric cancer cell AGS; therefore, the lower degree of differentiation, the stronger the cell invasion ability. Compared with control group, DS significantly reduced the invasion of all five types of cells in the experimental group, and it has the most notable inhibiting effect on BGC-823 cells, followed by the AGS, MGC-803, SGC-7901 and GES-1. It was determined that the strength of inhibition had no notable association with cell differentiation. A previous study demonstrated that matrix metalloproteinases secreted by tumor cells is the main component of degrading basement membrane and mesenchyme (15). The strength of inhibition by DS had a different order of cell lines for invasion and migration, which may be due to different abilities of these cells secreting matrix metalloproteinases and degrading extracellular matrix; in addition, this process could be influenced by DS in some degree for different cells, but the specific inhibition mechanism still requires further study.

$\beta$-catenin serves an important role in the process of invasion and migration of tumor cells, connecting adhesion molecules outside the cell to the frame within cells, through mediating the interaction of E-cad and $\beta$-catenin involved in the cell adhesion, migration and transfer process (16). $\beta$-catenin is highly expressed in gastric cancer cells AGS, HGC-27, MGC-803 and BGC-823. By contrast, it has a lower expression in normal gastric mucosa cells (17). Abnormal expression of E-cad is closely associated with tumor invasion, metastasis and diffusion (18). E-cad and $\beta$-catenin expression in different degrees of differentiation of gastric cancer cells, to a certain extent, could reflect the ability of invasion and migration.

$\beta$-catenin signaling pathway, also known as the Wnt signaling pathway, contains $\beta$-catenin, which has a key role in this pathway. It combines with the E-cad intracellular area, producing the $\beta$-catenin/E-cad complex, then connects to the actin cytoskeleton, mediates adhesion between cells, thus regulating the invasion and metastasis of tumor cells (19). The loss of combined E-cad leads to the accumulation of intracellular $\beta$-catenin, causing an increasing in $\beta$-catenin within nuclear localization in diffuse-gastric cancer, and at the same time an immunohistochemical staining study has demonstrated the reduction in E-cad on membrane (20). Accordingly, immunofluorescence staining was conducted to observe the expression changes; the effect of DS on MGC-803 is not particularly evident using CCK-8, therefore DS effective cells (BGC-823) was selected for further study. The results indicated that, with time, the expression of HIF- $1 \alpha$, Twist and N-cad in the control group cells gradually increased, whereas the E-cad expression gradually reduced. Simultaneously, it was considered that the $\beta$-catenin expression gradually increased, including in the nuclei; however, DS significantly reduced the expression of $\beta$-catenin in cell nucleus. As a necessary endogenous signal, Wnt signaling pathways can also directly control the ability of HIF-1 $\alpha$ to induce the occurrence of EMT (21); therefore, it was speculated that DS may use the Wnt pathway to inhibit the expression of HIF-1 $\alpha$.

In a hypoxic microenvironment, HIF-1 $\alpha$ overexpression in cancer cells accelerates tumor growth and metastasis by inducing tumor cells secretion of VEGF, regulating angiogenesis and promoting EMT $(22,23)$. A previous study confirmed that HIF-1 $\alpha$ induced tumor cell EMT through regulating the transcription factor Twist, which inhibits the expression of E-cad and promotes the expression of N-cad in hypoxia (24). Another study demonstrated that DS can inhibit the HIF-1 $\alpha$ expression on the greater momentum of the mice (5). In the present study, gastric cancer cell line BGC-823 was treated with DS and PBS, cultured in vitro in normoxia and hypoxia conditions, checked at various time points, observed for cell morphology changes. RT-PCR and western blot analysis were also conducted for further detection, the results demonstrated that DS have different degrees of inhibition on HIF- $1 \alpha$, Twist and $\mathrm{N}$-cad and $\beta$-catenin, as well as stimulation of E-cad expression; therefore, DS may affect Twist and E-cad through HIF-1 $\alpha$, inhibiting the occurrence of EMT.

In conclusion, DS has different degrees of inhibition on the proliferation of cell lines AGS, BGC-823, SGC-7901 and GES-1, which may be associated with the degree of differentiation. DS has a different degree of inhibition effect on migration and invasion ability of all five types of cells. Furthermore, DS may inhibit HIF-1 $\alpha$ through the Wnt signaling pathway, reducing the gene and protein level of Twist, increasing the 
epithelial marker E-cad and reducing the expression of mesenchymal marker $\mathrm{N}$-cad, thus inhibiting EMT of gastric cancer cell line BGC-823. In order to further study the anticancer mechanism of DS in human gastric cancer metastasis, experiments in vivo will be carried out in the subsequent tests.

\section{Acknowledgements}

Not applicable.

\section{Funding}

This study was supported by Ningxia Science and Technology Support Projects, West China First-Class Discipline Project in Basic Medical funded by Ningxia Medical University, The School of Basic Medicine at Ningxia Medical University and The Institute of Basic Medicine at Ningxia Medical University (grant no. 201-30181601).

\section{Availability of data and materials}

All data generated or analyzed during this study are included in this published article.

\section{Authors' contributions}

YX designed the experiment, supervised the study and confirmed the results. XJ performed the experiments and collected and analyzed the data. YH designed the experiments, confirmed the results and contributed to the writing of the manuscript. JW and XW performed part of the experiments. HW analyzed part of the data.

\section{Ethics approval and consent to participate}

Not applicable.

\section{Consent for publication}

Not applicable.

\section{Competing interests}

The authors declare that they have no competing interests.

\section{References}

1. Jemal A, Bray F, Center MM, Ferlay J, Ward E and Forman D: Global cancer statistics. CA-Cancer J Clin 61: 69-90, 2011.

2. Shen L, Shan YS, Hu HM, Price TJ, Sirohi B, Yeh KH, Yang YH, Sano T, Yang HK, Zhang X, et al: Management of gastric cancer in Asia: Resource-stratified guidelines. Lancet Oncol 14: e535-e547, 2013

3. Takagi T, Sakakura C, Kin S, Nakase Y, Fukuda K, Shimomura K, Ito T, Fujiyama J, Yamasaki J, Tsujimoto H, et al: Dextran sulfate suppresses cell adhesion and cell cycle progression of melanoma cells. Anticancer Res 25: 895-902, 2005.

4. Xu YY, Huang YN, Wang HH and Liu Y: Inhibition of the peritoneal metastasis of human gastric cancer cells by dextran sulphate in vivo and in vitro. Oncol Lett 11: 2384-2390, 2016.
5. Xu Y, Jin X, Huang Y, Dong J, Wang H, Wang X and Cao X: Inhibition of peritoneal metastasis of human gastric cancer cells by dextran sulphate through the reduction in HIF-1 $\alpha$ and ITG $\beta 1$ expression. Oncol Rep 35: 2624-2634, 2016.

6. Gurzu S, Turdean S, Kovecsi A, Contac AO and Jung I: Epithelial-mesenchymal, mesenchymal-epithelial, and endothelial-mesenchymal transitions in malignant tumors: An update. World J Clin Cases 3: 393-404, 2015.

7. Daly CS, Flemban A, Shafei M, Conway ME, Qualtrough D and Dean SJ: Hypoxia modulates the stem cell population and induces EMT in the MCF-10A breast epithelial cell line. Oncol Rep 39: 483-490, 2018.

8. Noman MZ, Messai Y, Carré T, Akalay I, Méron M, Janji B, Hasmim $\mathrm{M}$ and Chouaib S: Microenvironmental hypoxia orchestrating the cell stroma cross talk, tumor progression and antitumor response. Crit Rev Immunol 31: 357-377, 2011.

9. Bao B, Wang Z, Ali S, Kong D, Li Y, Ahmad A, Banerjee S, Azmi AS, Miele L and Sarkar FH: Notch-1 induces epithelial-mesenchymal transition consistent with cancer stem cell phenotype in pancreatic cancer cells. Cancer Lett 307: 26-36, 2011.

10. Goggins BJ, Chaney C, Radford-Smith GL, Horvat JC and Keely S: Hypoxia and integrin-mediated epithelial restitution during mucosal inflammation. Front Immunol 4: 272, 2013.

11. Peng Z, Wang CX, Fang EH, Wang GB and Tong Q: Role of epithelial-mesenchymal transition in gastric cancer initiation and progression. World J Gastroenterol 20: 5403-5410, 2014.

12. Fu F, Hu H, Yang S and Liang X: Effects of TIN2 on telomeres and chromosomes in the human gastric epithelial cell line GES-1. Oncol Lett 15: 5161-5166, 2018.

13. Goto A, Nishikawa J, Hideura E, Ogawa R, Nagao M, Sasaki S, Kawasato R, Hashimoto S, Okamoto T, Ogihara H, et al: Lymph node metastasis can be determined by just tumor depth and lymphovascular invasion in early gastric cancer patients after endoscopic submucosal dissection. Eur J Gastroenterol Hepatol 29: 1346-1350, 2017.

14. Hou XH: The expression of HORMAD2 and the effects of it on proliferation and apoptosis on gastric cancer cell (D). Lanzhou Univ, 2015.

15. Weili Xu: The effect of lysine (K)-specific demethylase $1 \mathrm{~A}$ on invasion and metastasis of gastric cancer and its associated mechanism (D). Central South Univ, 2014.

16. Zheng L, Hu X, Huang Y, Xu G, Yang J and Li L: In vivo bioengineered ovarian tumors based on collagen, matrigel, alginate and agarose hydrogels: A comparative study. Biomed Mater 10: 015016, 2015

17. Cheng XX, Wang ZC, Chen XY, Sun Y, Kong QY, Liu J, Gao X, Guan HW and Li H: Frequent loss of membranous E-cadherin in gastric cancers: A cross-talk with Wnt in determining the fate of beta-catenin. Clin Exp Metastasis 22: 85-93, 2005.

18. Dong Li: Expression of $\beta$-catenin-the Central Regulation Molecule of the Wnt Signaling Pathways in gastric cancer and its significance (D). Nanchang Univ, 2014.

19. Zhang Z, Bu X, Chen H, Wang Q and Sha W: Bmi-1 promotes the invasion and migration of colon cancer stem cells through the downregulation of E-cadherin. Int J Mol Med 38: 1199-1207, 2016.

20. Huelsken $\mathrm{J}$ and Behrens J: The Wnt signalling pathway. J Cell Sci 115: 3977-3978, 2002.

21. Kalluri R and Weinberg R: The basics of epithelial-mesenchymal transition. J Clin Invest 119: 1420-1428, 2009.

22. Ashaie MA and Chowdhury EH: Cadherins: The superfamily critically involved in breast cancer. Curr Pharm Des 22: 616-638, 2016.

23. Zhang X, Liu G, Kang Y, Dong Z, Qian Q and Ma X: N-cadherin expression is associated with acquisition of EMT phenotype and with enhanced invasion in erlotinib-resistant lung cancer cell lines. PLoS One 8: e57692, 2013.

24. Wu S, Luo Z, Yu PJ, Xie H and He YW: Suberoylanilide hydroxamic acid (SAHA) promotes the epithelial mesenchymal transition of triple negative breast cancer cells via HDAC8/FOXA1 signals. Biol Chem 397: 75-83, 2016.

This work is licensed under a Creative Commons Attribution-NonCommercial-NoDerivatives 4.0 International (CC BY-NC-ND 4.0) License. 